\title{
Effect of bladder volume on radiation doses to organs at risk and tumor in cervical cancer during image-guided adaptive brachytherapy and treatment outcome analysis
}

\author{
ZHIYUAN XU ${ }^{1,2}$, LI YANG ${ }^{1,3}$, LINGYU MA ${ }^{1}$, QIN LIU ${ }^{1}$, AMY TY CHANG ${ }^{1,4}$, YONG ZHOU ${ }^{1}$, \\ CHENG ZHOU $^{2,5}$, FENG-MING KONG ${ }^{1,6,7}$ and LONGHUA CHEN ${ }^{2}$
}

\begin{abstract}
${ }^{1}$ Oncology Medical Center, The University of Hong Kong-Shenzhen Hospital, Shenzhen, Guangdong 518053;
${ }^{2}$ Department of Radiation Oncology, Nanfang Hospital, Southern Medical University, Guangzhou, Guangdong 510515;

${ }^{3}$ Department of Pathology, Zhujiang Hospital, Southern Medical University, Guangzhou, Guangdong 510282;

${ }^{4}$ Department of Clinical Oncology, Hong Kong Sanatorium and Hospital, Hong Kong 999077, Hong Kong, SAR;

${ }^{5}$ Department of Oncology, Guangdong Sanjiu Brain Hospital, Guangzhou, Guangdong 510510;

${ }^{6}$ Department of Clinical Oncology, The University of Hong Kong, Hong Kong 999077, Hong Kong, SAR, P.R. China;

${ }^{7}$ Department of Radiation Oncology, Case Western Reserve University School of Medicine, Cleveland, OH 44106, USA
\end{abstract}

Received March 25, 2021; Accepted August 26, 2021

DOI: $10.3892 / \mathrm{mco} .2021 .2420$

\begin{abstract}
There is no consensus in clinical practice on the optimal bladder volume during brachytherapy. The present study aimed to assess the effect of bladder volume on radiation dose to organs at risk and tumor in cervical cancer during image-guided adaptive brachytherapy and clinical outcome. The retrospective study included patients treated at University of Hong Kong-Shenzhen Hospital between January 2015 and July 2019. Patients with International Federation of Gynecology and Obstetrics (2009) stage IB1-IVB (retroperitoneal lymph nodes metastasis only) cervical cancer treated by external beam radiotherapy with concurrent cisplatin followed by brachytherapy were assessed. A total of 421 brachytherapy insertions were analyzed. Every 83 and $90 \mathrm{~cm}^{3}$ (cc) increase in bladder volume led to an incremental raise of $1 \mathrm{~Gy}$ in bladder wall minimum dose received by the most irradiated 1 and $2 \mathrm{cc}$ volumes (D1 and D2cc) of the bladder wall, respectively. An increase in bladder volume was associated with increased D1 and D2cc of bladder (both $\mathrm{P}<0.001$, respectively) and rectal wall $(\mathrm{P}=0.150$ and $\mathrm{P}=0.084$, respectively), and decreased $\mathrm{D} 1 \mathrm{cc}(\mathrm{P}=0.003)$ and $\mathrm{D} 2 \mathrm{cc}(\mathrm{P}=0.001)$ of sigmoid wall, the maximum doses to the most minimally exposed 90 (D90) and 95\% (D95)
\end{abstract}

Correspondence to: Dr Longhua Chen, Department of Radiation Oncology, Nanfang Hospital, Southern Medical University, 1838 Guangzhou Avenue North, Guangzhou, Guangdong 510515, P.R. China

E-mail: chen1hsmu@126.com

Key words: cervical cancer, brachytherapy, bladder volume, radiotherapy dose, overall survival of the high risk-clinical target volume (HR-CTV; D90, $\mathrm{P}=0.010 ; \mathrm{D} 95, \mathrm{P}=0.006$ ). Patients with cumulative HR-CTV D90 $\leq 89.6$ Gy had shorter median overall survival (OS) than those with cumulative HR-CTV D90>89.6 Gy (42.1 months vs. not reached, $\mathrm{P}=0.001)$. Patients with grade 2 acute urinary toxicity had significantly higher cumulative bladder wall D2cc than those with acute urinary toxicity<grade 2 ( $86.7 \pm 3.7$ vs. $78.5 \pm 7.9 \mathrm{~Gy} ; \mathrm{P}=0.001)$. As the bladder volume increased, the dose to the bladder and rectal wall increase and dose to the sigmoid colon wall and HR-CTV decrease based on intracavitary brachytherapy. The higher dose of HR-CTV predicted better OS and the higher dose received by the bladder wall was associated with more grade 2 acute urinary toxicity.

\section{Introduction}

Cervical cancer is a major health burden worldwide; there were an estimated 13,170 new cases of cervical cancer and 4,250 deaths in 2019 (1). The standard treatment for localized inoperable cervical cancer is external beam radiotherapy (EBRT) with concurrent cisplatin followed by brachytherapy (2). Dose optimization using intracavitary applicators has been shown to significantly decrease doses to organs at risk (OARs) and morbidity (3). OARs, such as bladder, rectum and sigmoid colon, are dose-limiting as radiation above certain levels can cause further complications. Brachytherapy induces enteritis and cystitis due to irradiation to pelvic OARs. Therefore, it is crucial to optimize the dose to OARs to minimize the risk of complications (4-6). The volume and position of OARs in the pelvis affect the position of the tumor and the dose to both OARs and tumor. Bladder volume is one of the most important variables during brachytherapy insertions because of the notable changes in bladder contents. The effect of bladder volume on doses to OARs and 
tumor is unclear. There is no consensus in clinical practice on the optimal bladder volume during brachytherapy (7-9). Certain studies have suggested that smaller bladder volume is better to achieve a lower dose to OARs (5) while others have shown that full bladder is non-inferior with regard to bladder dose $(7,9)$. Another study suggested that bladder volume exhibits different dosimetric effects on different OARs (10). Various bladder filling protocols have been recommended with limited evidence, including empty bladder with indwelling Foley's catheter (11), a known limited filling status (50 cc) (12) and full bladder (7). The International Commission on Radiation Units and Measurements (ICRU) 89 report had no recommendations on bladder volume status (full, empty or other) during pelvic intracavitary brachytherapy (13). Ongoing clinical study Embrace II (14) by European Brachytherapy Group-European Society for Radiotherapy and Oncology Gynecology (GEC-ESTRO GYN) working group has not specified bladder volume control during brachytherapy. To the best of our knowledge, few studies have assessed the effect of bladder volume on OAR dose and tumor during image-guided adaptive brachytherapy for cervical cancer or the treatment results.

The present study aimed to determine the optimal bladder volume to guide clinical practice by investigating the effect of bladder volume on cervical cancer OARs and primary tumor during image-guided adaptive brachytherapy and its impact on treatment outcome.

\section{Materials and methods}

Patients. The present study was approved by the Medical Ethics Committee of the University of Hong Kong-Shenzhen Hospital (Shenzhen, China). All 109 patients with cervical cancer who received radical radiotherapy at Oncology Medical Center of the University of Hong Kong-Shenzhen Hospital between January 2015 and July 2019 were included in the retrospective study and relevant information was obtained from medical records. The inclusion criteria were as follows: i) age $\geq 18$ years, ii) ECOG performance status $\leq 2$, iii) pathology-confirmed, International Federation of Gynecology and Obstetrics (FIGO; 2009) (15) stage IB1-IVB (retroperitoneal lymph nodes metastasis only) cervical cancer, and iv) patients were treated with EBRT with concurrent cisplatin followed by image-guided adaptive brachytherapy. Patients were excluded if they had a history of pelvic surgery or irradiation or recurrent cervical cancer.

Brachytherapy applicator insertion procedure. Intracavitary brachytherapy was performed 2-3 weeks after initiation of EBRT. Brachytherapy applicators were inserted under general anesthesia. A Foley's catheter was inserted and $150 \mathrm{cc}$ normal saline was injected into bladder after general anesthesia. The intrauterine tandem was inserted under ultrasound guidance. The tandem length with stopper was selected based on the length of the uterine cavity. Ring or ovoid shaped applicators were selected based on disease extent and vaginal distensibility. Following the completion of applicator insertion, the Foley's catheter was kept open with connection to a urine bag until the end of each brachytherapy session. The
Foley's catheter was removed after each brachytherapy session. Simulation, delineation of bladder volume/wall and treatment were performed in the empty bladder state.

Imaging and contouring. All patients underwent CT simulation with $1 \mathrm{~mm}$ axial image slices in treatment position from the 10th thoracic vertebra to the upper third of the femur. The CT images were transferred to Varian eclipse treatment planning system, (version 15.0; Varian Medical Systems). HR-CTV and OARs (bladder, rectum and sigmoid colon) were delineated on the CT image (Fig. 1) with reference to available information (including gynecological examination, magnetic resonance imaging at the time of diagnosis and before brachytherapy) by a radiation oncologist according to the GEC-ESTRO GYN working group recommendations (2) and were confirmed by a radiation oncologist consultant. OARs were defined by Radiation Therapy Oncology Group Consensus Panel Atlas (16). Rectum contouring started inferiorly from the lowest level of the ischial tuberosities (right or left) and ended superiorly before the rectum lost its round shape in the axial plane and connected anteriorly with the sigmoid. The volume of OARs and HR-CTV were obtained from CT simulation scan by the Varian eclipse treatment planning system (version 15.0). Bladder, rectum and sigmoid colon volume comprised the entire volume, including the wall and contents of bladder, rectum and sigmoid colon.

Treatment planning. EBRT was delivered on a $6 \mathrm{MV}$ linear accelerator; prescription dose was $45 \mathrm{~Gy}$ in 25 fractions to whole pelvis with a simultaneous integrated boost of 55.0-57.5 Gy to pelvic or retroperitoneal metastatic lymph nodes (Fig. 2). Concurrent cisplatin at $40 \mathrm{mg} / \mathrm{m}^{2}$ was given weekly during EBRT. All 109 patients received the same dose of external beam therapy but different doses of brachytherapy in the treatment planning section. The image-guided brachytherapy plan was constructed using the Varian eclipse treatment planning system (version 15.0). The prescription dose for each high-dose-rate (HDR) brachytherapy was 6-7 Gy to HR-CTV, four times in total. Equivalent dose (EQD2) (17) was calculated for HDR treatments using the linear quadratic model normalized to $2 \mathrm{~Gy} /$ fraction as follows: $\mathrm{EQD} 2=\mathrm{n} \times \mathrm{d} \times \frac{\mathrm{d}+\alpha / \beta}{2+\alpha / \beta}$, where $\mathrm{n}$ represents radiotherapy session number, $\mathrm{d}$ represents the dose and $\alpha / \beta$ ratio (18) represents radiotherapy sensitivity of tumor and normal tissue. The full course treatment plan, including external radiotherapy and brachytherapy evaluation criteria of OARs for all patients, were as follows: Rectum, D2cc<75 Gy (EQD2); sigmoid colon, D2cc $<75$ Gy (EQD2) and bladder D2cc $<90$ Gy (EQD2). Dose prescription of HR-CTV D90 was as follows: Stage IB-IIIA, $>84$ Gy (EQD2) and $\geq$ stage IIIB, $>90$ Gy (EQD2). Dose constraint of OARs had a higher priority than HR-CTV. Dose of external irradiation and brachytherapy were converted to EQD2, then HR-CTV D90 and D2cc of rectum, sigmoid and bladder during external radiotherapy and brachytherapy were calculated using deformable registration by medical imaging software version 6.9.5, MIM Software Inc. Doses to HR-CTV and the D2 and Dlcc of bladder, rectal and sigmoid wall were obtained from dose-volume histograms (DVHs) automatically generated by the Varian eclipse treatment planning system (version 15.0). Toxicity during concurrent chemoradiotherapy (CCRT) 
Table I. Patient and clinicopathological characteristics.

\begin{tabular}{lc}
\hline Characteristic & Number $(\%), \mathrm{n}=109$ \\
\hline Median age (range), years & $53.00(33.00-77.00)$ \\
Median BMI (range) & $22.67(15.87-29.80)$ \\
ECOG score & \\
$0-1$ & $97.00(89.00)$ \\
2 & $12.00(11.00)$ \\
Histology & \\
Squamous cell carcinoma & $101.00(92.70)$ \\
Adenocarcinoma & $4.00(3.70)$ \\
Other & $4.00(3.70)$ \\
FIGO stage & \\
IB1 & $5.00(4.60)$ \\
IB2 & $2.00(1.80)$ \\
IIA1 & $4.00(3.70)$ \\
IIA2 & $9.00(8.30)$ \\
IIB & $38.00(34.90)$ \\
IIIA & $4.00(3.70)$ \\
IIIB & $39.00(35.80)$ \\
IVA & $3.00(2.80)$ \\
IVB & $5.00(4.60)$ \\
Brachytherapy insertion & \\
1 & \\
2 & \\
Ring & \\
Ovoid & \\
\hline
\end{tabular}

BMI, body mass index; ECOG, Eastern Cooperative Oncology Group; FIGO, International Federation of Gynecology and Obstetrics.

was graded according to Common Terminology Criteria for Adverse Events 4.03 criteria (19).

Statistical analysis. Data analysis was performed using SPSS version 19.0 for Windows (IBM, Corp.), GraphPad prism 8 (GraphPad Software, Inc.) and R Programming Language version 3.6.1 (R Studio, Inc.). Clinicopathological and treatment-associated characteristics were assessed using descriptive statistics. Paired t-test (mean \pm SD) was used to compare means between two groups. Association between bladder volume and dose to OARs and HR-CTV were analyzed using linear regression model. Cumulative HR-CTV D90 represented the total dose received by HR-CTV during the whole course of RT and was defined as the integrated dose to $90 \%$ of HR-CTV including both EBRT and brachytherapy. Local failure was defined as local tumor residual at the end of treatment or local recurrence during follow up.
Table II. Linear regression model of factors associated with bladder volume during brachytherapy.

\begin{tabular}{lcccr}
\hline & \multicolumn{3}{c}{$95 \%$ CI limit } \\
\cline { 3 - 4 } Factor & OR & Lower & Upper & P-value \\
\hline Rectum volume & 0.222 & -0.018 & 0.462 & 0.070 \\
Sigmoid volume & 0.190 & 0.102 & 0.278 & $<0.001$ \\
HR-CTV volume & 0.168 & -0.053 & 0.389 & 0.136 \\
Corpus angle & 0.135 & -0.058 & 0.327 & 0.170 \\
Age & 0.105 & -0.185 & 0.395 & 0.477 \\
BMI & 0.087 & -0.850 & 1.025 & 0.855
\end{tabular}

OR, odds ratio; CI confidence interval; HR-CTV, high risk-clinical target volume; BMI, body mass index.

Local control was defined as no local residual at the end of treatment and no local recurrence during follow up. Overall survival (OS) was the time from the start of EBRT to the date of death from any cause or the last confirmed date of survival. Local control and OS of all patients were based on both brachytherapy and external beam therapy. Receiver operating characteristic (ROC) curve was used to select cutoff values of cumulative HR-CTV D90 predicting local failure. Survival was evaluated using Kaplan-Meier curves with the log-rank test and Cox proportional hazard model. Bilateral $\mathrm{P}<0.05$ was considered to indicate a statistically significant difference.

\section{Results}

Patient characteristics. A total of consecutive 109 patients with 421 intracavitary brachytherapy insertions were included. The median age was 53 years (range, 33-77 years). The FIGO (2009) stage distribution was 7 (6.4), 51 (46.8), $43(39.4 \%)$ and $8(7.3 \%)$ for stage I-III and IV, respectively. A total of 101 patients $(92.7 \%)$ had squamous cell carcinoma. A total of 99 patients $(90.8 \%$ ) completed the scheduled four brachytherapy insertions. Patient and clinicopathological characteristics are presented in Table I. A total of two patients refused to continue brachytherapy due to uterine perforation and vaginal injury during the last brachytherapy; eight patients changed to stereotactic body radiation therapy or external radiation boost due to large residual volume following external radiotherapy with expected poor dose coverage by intracavity brachytherapy.

Bladder volume during brachytherapy. The Foley's catheter was kept open with connection to a urine bag during brachytherapy. The median volume of bladder, rectum, sigmoid colon and HR-CTV of all 421 brachytherapy insertions on simulation CT was 60.1 (range, 13.5-318.1), 28.3 (8.6-104.0), 49.6 (6.0-274.6) and 22.6 (5.6-108.1 cc), respectively. Linear regression model, including volumes of rectum, sigmoid colon and HR-CTV, corpus angle, age and body mass index (BMI), showed that sigmoid [odds ratio (OR), 
Table III. Change in bladder volume between brachytherapy insertions (paired t-test).

\begin{tabular}{|c|c|c|c|c|c|c|}
\hline Pair & & $\mathrm{N}$ & $\begin{array}{l}\text { Bladder volume } \\
(\text { mean } \pm \mathrm{SD}), \mathrm{cc}\end{array}$ & $\begin{array}{l}\text { Change in bladder volume } \\
\quad(\text { mean } \pm \mathrm{SD}), \text { cc }\end{array}$ & $\mathrm{t}$ & P-value \\
\hline \multirow[t]{2}{*}{1} & 2nd insertion & 108 & $67.5 \pm 36.9$ & $1.9 \pm 34.6$ & 0.582 & 0.562 \\
\hline & 1st insertion & 108 & $65.5 \pm 24.8$ & & & \\
\hline \multirow[t]{2}{*}{2} & 3rd insertion & 105 & $65.8 \pm 29.8$ & $0.9 \pm 30.3$ & 0.302 & 0.763 \\
\hline & 1st insertion & 105 & $64.9 \pm 24.9$ & & & \\
\hline \multirow[t]{2}{*}{3} & 4th insertion & 99 & $73.7 \pm 37.7$ & $8.6 \pm 34.7$ & 2.472 & 0.015 \\
\hline & 1st insertion & 99 & $65.1 \pm 24.8$ & & & \\
\hline
\end{tabular}

$\mathrm{SD}$, standard deviation; cc, cubic centimeter.

Table IV. Cox regression analysis of factors associated with OS.

\begin{tabular}{|c|c|c|c|c|}
\hline \multirow[b]{2}{*}{ Factor } & \multirow[b]{2}{*}{ HR } & \multicolumn{2}{|c|}{$95 \%$ CI limit } & \multirow[b]{2}{*}{ P-value } \\
\hline & & Lower & Upper & \\
\hline Age $(<60 / \geq 60$ years $)$ & 2.277 & 0.730 & 7.108 & 0.156 \\
\hline $\operatorname{ECOG}(0-1 / 2)$ & 0.541 & 0.173 & 1.692 & 0.291 \\
\hline FIGO stage (I-II/III-IV) & 0.897 & 0.353 & 2.276 & 0.818 \\
\hline Hemoglobin $(<110 / \geq 110 \mathrm{~g} / \mathrm{l})$ & 2.271 & 0.933 & 5.532 & 0.071 \\
\hline Concurrent chemotherapy cycles $(0-4 / 5-6)$ & 3.252 & 1.209 & 8.748 & 0.020 \\
\hline Cumulative HR-CTV D90 & 0.940 & 0.892 & 0.990 & 0.019 \\
\hline
\end{tabular}

HR, hazard ratio; CI, confidence interval; ECOG, Eastern Cooperative Oncology Group; FIGO, International Federation of Gynecology and Obstetrics; HR-CTV, high risk-clinical target volume.

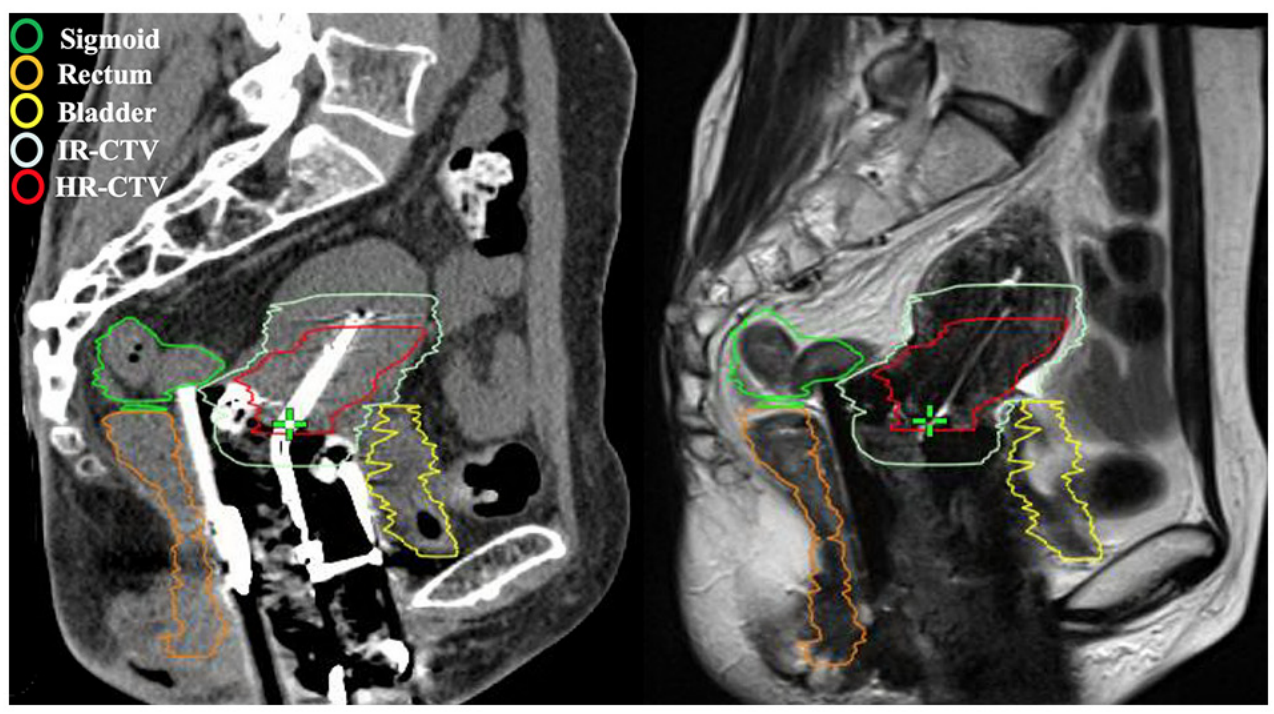

Figure 1. Diagram of a typical patient with cervical cancer showing organs at risk and targets. HR-CTV, high risk-clinical target volume. IR-CTV, intermediate risk-clinical target volume.

$0.190 ; 95 \% \mathrm{CI}, 0.102-0.278 ; \mathrm{P}<0.001]$ and rectum volume (OR, 0.222; 95\% CI, $-0.018-0.462 ; \mathrm{P}=0.070)$ were associated with bladder volume during brachytherapy. HR-CTV volume, corpus angle, age and BMI did not affect bladder volume during brachytherapy (Table II). With the increase of brachytherapy insertion time, paired t-test (Table III) 


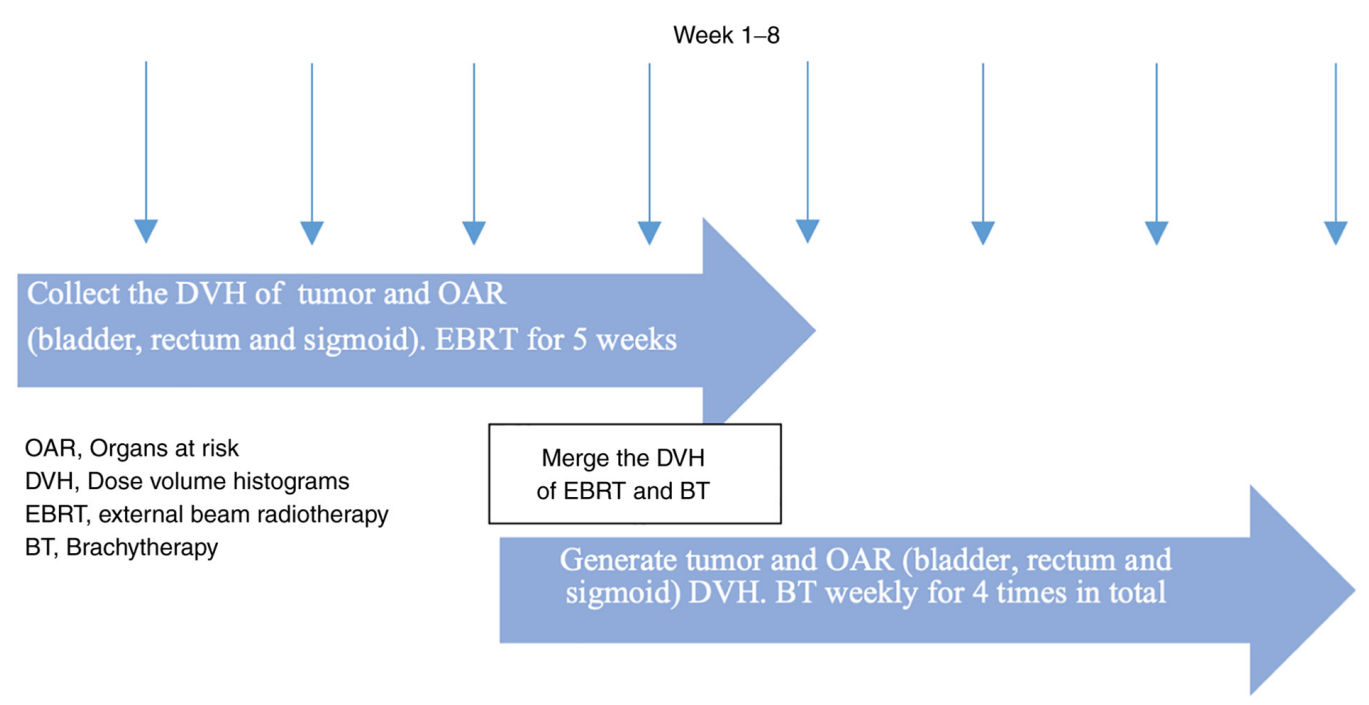

Figure 2. Radiotherapy delivery to all organs and tumor.

showed that bladder volume at the fourth insertion increased significantly compared with the first insertion (mean $\pm \mathrm{SD}$; $73.7 \pm 37.7$ vs. $65.1 \pm 24.8 \mathrm{cc} ; \mathrm{P}=0.015)$.

Effect of bladder volume on dose to OARs and tumor (HR-CTV). Linear regression analysis showed that bladder volume was associated significantly and linearly with D1 ( R, 0.315; P<0.001) and D2 $\mathrm{cc}(\mathrm{R}, 0.346 ; \mathrm{P}<0.001)$ of the bladder wall (data not shown). There was a 1 Gy increase in D1 and D2cc with every 83 or $90 \mathrm{cc}$ increase in bladder volume, respectively. Linear regression models were repeatedly constructed to evaluate the effect of bladder volume on dose to OARs and tumor (HR-CTV) by adjusting confounding factors, including rectum, sigmoid and HR-CTV volume, corpus angle, applicator length and BMI. With increasing bladder volume, D1cc (OR, 0.012; 95\% CI, 0.009-0.016; P<0.001) and D2cc (OR, 0.012; 95\% CI, 0.009-0.015; $\mathrm{P}<0.001)$ of bladder wall increased; D1 (OR, 0.002; 95\% CI, $-0.001-0.005 ; \mathrm{P}=0.150)$ and $\mathrm{D} 2 \mathrm{cc}(\mathrm{OR}, 0.002 ; 95 \% \mathrm{CI}$, $0.000-0.004 ; \mathrm{P}=0.084)$ of the rectal wall also increased. However, the D1 (OR, -0.005; 95\% CI, -0.008-0.002; $\mathrm{P}=0.003$ ) and D2cc (OR, -0.004; 95\% CI, -0.007-0.002; P=0.001) of sigmoid wall and HR-CTV D90 (OR, -0.005; 95\% CI, -0.009-0.001; $\mathrm{P}=0.010)$ and $\mathrm{D} 95(\mathrm{OR},-0.005 ; 95 \% \mathrm{CI}$, $-0.009-0.001 ; \mathrm{P}=0.006)$ decreased with increased bladder volume. These data were based on intracavitary brachytherapy. From these linear regression models, HR-CTV volume affected $\mathrm{D} 1$ and $\mathrm{D} 2 \mathrm{cc}$ of rectal ( $\mathrm{P}=0.058$ and 0.035 , respectively) and sigmoid wall ( $\mathrm{P}=0.015$ and 0.002 , respectively) and HR-CTV D90 and D95 (both $\mathrm{P}<0.001$ ); applicator length affected the D1 and D2cc of rectal (both $\mathrm{P}=0.012$ ) and sigmoid wall (both $\mathrm{P}<0.001$ ); and BMI affected D1 and D2cc (both $\mathrm{P}=0.001$ ) of bladder wall; corpus angle affected D1 and D2cc of rectal (both $\mathrm{P}<0.001)$ and sigmoid wall $(\mathrm{P}=0.056$ and 0.015 , respectively) and HR-CTV D90 and D95 (both P<0.001).

Effect of HR-CTV cumulative dose on local failure and $O S$. The median follow-up time was 28 months (range, 3-59 months); the median OS for the entire patient population was 54.0 months (95\% CI, 36.3-71.7 months).

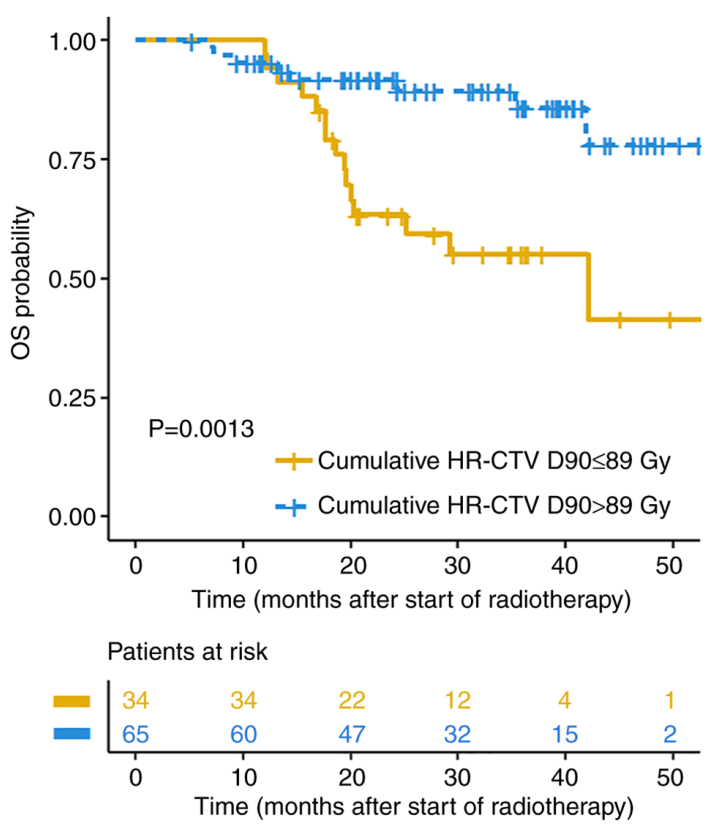

Figure 3. Overall survival curve by Kaplan-Meier. Patients with cumulative HR-CTV D90 $\leq 89.6$ Gy had shorter OS than those with cumulative HR-CTV D90>89.6 Gy (median OS, 42.1 months vs. not reached; $\mathrm{P}=0.001$ ). HR-CTV, high risk-clinical target volume; OS, overall survival.

Among the 99 patients who completed all four brachytherapy insertions, 14 experienced local failure. Patients with local failure had significantly lower cumulative HR-CTV D90 than those without local failure (mean \pm SD; $89.5 \pm 9.3$ vs. $97.0 \pm 12.4 \mathrm{~Gy} ; \mathrm{P}=0.032$ ). The cut-off value of cumulative HR-CTV D90 to predict local control on ROC analysis was $89.6 \mathrm{~Gy}$ with area under curve $=0.702$, sensitivity $=70.6 \%$ and specificity $=64.3 \%(\mathrm{P}=0.016)$. Patients with cumulative HR-CTV D90 $\leq 89.6$ Gy had shorter OS than those with cumulative HR-CTV D90>89.6 Gy (median OS, 42.1 months vs. not reached; $\mathrm{P}=0.001$; Fig. 3). Cox multivariate analysis demonstrated that higher cumulative HR-CTV D90 [hazard ratio (HR), 0.940; 95\% CI, $0.892-0.990 ; \mathrm{P}=0.019]$ was associated with better OS after 
adjusting age, ECOG, FIGO stage, hemoglobin before treatment and concurrent chemotherapy cycles (Table IV).

Effect of bladder volume on treatment-associated toxicity. Toxicity during CCRT was graded according to CTCAE 4.03 criteria (19). Cumulative bladder wall D2cc is defined as the integrated dose to the highest irradiated $2 \mathrm{cc}$ area of bladder wall, including both EBRT and brachytherapy phase. Patients with grade 2 acute urinary toxicity had significantly higher cumulative bladder wall D2cc than those with acute urinary toxicity <grade 2 (mean $\pm \mathrm{SD} ; 86.7 \pm 3.7$ vs. $78.5 \pm 7.9 \mathrm{~Gy}$; $\mathrm{P}=0.001$; data not shown). Only one patient experienced grade 3 late intestinal toxicity.

\section{Discussion}

The present study analyzed the effect of bladder volume on OARs and HR-CTV radiation dose and associated treatment results during brachytherapy for cervical cancer and aimed to provide recommendations for bladder volume control during brachytherapy to decrease the dose to normal tissue while optimizing the dose to the primary tumor. The present study found as bladder volume increased, the dose to the bladder and rectal walls increased and the dose to sigmoid wall and HR-CTV decreased. A higher priority was set for OARs to avoid serious complications and results were based on intracavitary, rather than interstitial, brachytherapy (20). Higher doses of HR-CTV predicted better OS throughout the course of radiotherapy and higher dose to the bladder wall was associated with more grade 2 acute urotoxicity. From a clinical perspective, it is therefore better to minimize bladder volume during brachytherapy for cervical cancer.

Based on previous studies $(8,12,21)$, there is no consistent recommendation for bladder volume control during brachytherapy. Sharma et al (21) showed that D2cc of the bladder increase with bladder volume; bladder volume $\leq 70 \mathrm{cc}$ proved better for achieving lower radiation dose of bladder. Siavashpour et al (8) showed that bladder D0.1 and D2cc increase significantly with bladder volume and concluded that bladder volume $<70 \mathrm{cc}$ might be a better choice than bladder volume $>70 \mathrm{cc}$. Mahantshetty et al (12) suggested that bladder filling protocol with 50 or $100 \mathrm{cc}$ was well tolerated and achieved a reasonably reproducible bladder volume during cervical brachytherapy. The present study showed that bladder volume was significantly and linearly associated with D1 and D2cc of the bladder wall and there was a $1 \mathrm{~Gy}$ increase in D1 or D2cc with every 83 or $90 \mathrm{cc}$ increase in bladder volume, respectively. This means that the smaller the bladder volume, the lower the bladder wall dose.

Sharma et al (21) showed that the dose to rectum increases with bladder volume but decreases after bladder volume reaches $110 \mathrm{cc}$; sigmoid DVH parameters followed a similar trend as that of the rectum. Siavashpour et al (8) showed that the rectum dose decreases with bladder volume $>140 \mathrm{cc}$ and sigmoid dose decreases with bladder volumes $<75 \mathrm{cc}$; however, for bladder volume $>75 \mathrm{cc}$, the sigmoid dose increased. The D2cc of bladder and rectum were higher for longer applicator lengths than shorter ones (8). Mahantshetty et al (12) found that rectal and sigmoid doses are not significantly affected by bladder volume. Harmon et al (7) showed that sigmoid D2 and D1cc are significantly decreased in full bladder plans; the rectum shows no significant difference in D2 and D0.1cc between different bladder volumes. The present data showed that D1 and D2cc of rectal wall increased with bladder volume. However, D1 and D2cc of sigmoid decreased with increased bladder volume. The different results between these studies may be due to different methods of bladder volume control and grouping. The present study showed HR-CTV volume affected the D1 and D2cc of rectal and sigmoid wall and HR-CTV D90 and D95; and applicator length affected the D1 and D2cc of rectal and sigmoid wall. Applicator length may affect doses of OARs through the position change of bladder and rectum. BMI affected the D1 and D2cc of bladder wall; corpus angle affected D1 and D2cc of rectal and sigmoid wall and HR-CTV D90 and D95. Siavashpour et al (10) demonstrated that empty bladder status may decrease the dose to rectum and sigmoid with tandems $>4 \mathrm{~cm}$, which was similar to results of the present study. Several studies have demonstrated significant increase in small bowel dose with decreased bladder volume $(7,9,12)$. Nevertheless, the small bowel dose received may not be a true reflection from the dose estimated by the radiotherapy plan because of the changes in the position of the small intestine during treatment $(9,22,23)$.

The present study demonstrated that bladder volume at the fourth insertion was significantly increased compared with the first insertion. However, the Foley's catheter was kept open during CT simulation for all patients. The present study showed that sigmoid and rectum volume were associated with bladder volume during brachytherapy. HR-CTV volume, corpus angle, age and BMI did not affect bladder volume during brachytherapy. Statistical analysis showed that BMI affected D1 and D2cc of bladder wall but not bladder volume. This may be because obesity increases the difficulty and affects the accuracy of application insertion and subcutaneous fat may shorten the distance between the bladder and tumor, resulting in an increased radiation dose to the bladder wall without affecting the bladder volume. In addition, although the catheter was kept open, bladder volumes varied greatly. Potential causes include poor drainage of the catheter, decreased bladder contraction caused by radiotherapy and general anesthesia affecting bladder emptying. Lee et al (24) showed that interfractional dosimetric variation for both target and OARs resulted in a change of treatment plan in $6 \%$ of cases. Therefore, controlling bladder volume is an important clinical issue.

The present study showed that cumulative HR-CTV D90 in patients with local failure was significantly lower than that in patients without local failure. Local control and OS were lower when cumulative HR-CTV D90 $\leq 89.6$ Gy. Patients with grade 2 acute urinary toxicity exhibited significantly higher cumulative bladder wall D2cc than those with acute urinary toxicity $<$ grade 2 , which is similar to other studies $(4,6)$. The present study analyzed both treatment outcomes and effect of bladder volume, filling gaps in other studies. However, the study was limited by the retrospective nature of the design. The catheter remained open during brachytherapy without bladder volume control procedures; bladder volume control will be the focus of future research. Interstitial brachytherapy has 
notable dosimetric advantages for patients with cervical cancer with asymmetrical growth or large mass which makes it difficult to achieve ideal dose distribution via intracavitary brachytherapy (20). Magnetic resonance image-guided brachytherapy or interstitial brachytherapy provide more accurate tumor and OAR delineation and dose assessment $(20,25,26)$, which is also a future research direction.

In conclusion, restricting bladder volume during brachytherapy may decrease doses to the bladder and rectal wall. Higher dose to the bladder wall was associated with more grade 2 acute urinary toxicity. Moreover, smaller bladder volume may increase the dose to HR-CTV; patients with cumulative HR-CTV D90>89.6 Gy have better local control and OS.

\section{Acknowledgements}

Not applicable.

\section{Funding}

The present study was supported in part by Health Commission of Guangdong Province (grant no. B2020100); High Level-Hospital Program, Health Commission of Guangdong Province (grant no. HKUSZH201902031); Shenzhen Healthcare Research Project, China (grant no. SZXJ2018003); Shenzhen Key Medical Discipline Construction Fund (grant no. SZXK014) and Shenzhen Science and Technology Program (grant no. KQTD20180411185028798).

\section{Availability of data and materials}

The datasets used and/or analyzed during the current study are available from the corresponding author on reasonable request.

\section{Authors' contributions}

LC, ZX, ATC, YZ, CZ, FMK and QL designed the study. ZX, ATC, YZ, CZ, FMK, QL, LM and LY performed the literature review, analyzed data and wrote the manuscript. ZX, LY, ATC, YZ, CZ, FMK and LC guaranteed the integrity of the study. ZX, LY, YZ, QL and CZ, LM collected data. LC, ZX, LY, QL, ATC and FMK, LM revised the manuscript. All authors have read and approved the final version of the manuscript. ZX and LY confirm the authenticity of all the raw data.

\section{Ethics approval and consent to participate}

The present study was approved by the Medical Ethics Committee of the University of Hong Kong-Shenzhen Hospital (approval no. hkuszh2019119).

\section{Patient consent for publication}

Not applicable.

\section{Competing interests}

The authors declare that they have no competing interests.

\section{References}

1. Siegel RL, Miller KD and Jemal A: Cancer statistics, 2019. CA Cancer J Clin 69: 7-34, 2019.

2. Haie-Meder C, Pötter R, Van Limbergen E, Briot E, De Brabandere M, Dimopoulos J, Dumas I, Hellebust TP, Kirisits C, Lang S, et al: Recommendations from gynaecological (GYN) GEC-ESTRO working group (I): Concepts and terms in 3D image based 3D treatment planning in cervix cancer brachytherapy with emphasis on MRI assessment of GTV and CTV. Radiother Oncol 74: 235-245, 2005.

3. Charra-Brunaud C, Harter V, Delannes M, Haie-Meder C, Quetin P, Kerr C, Castelain B, Thomas L and Peiffert D: Impact of 3D image-based PDR brachytherapy on outcome of patients treated for cervix carcinoma in France: Results of the French STIC prospective study. Radiother Oncol 103: 305-313, 2012.

4. Manea E, Escande A, Bockel S, Khettab M, Dumas I, Lazarescu I, Fumagalli I, Morice P, Deutsch E, Haie-Meder C and Chargari C: Risk of late urinary complications following image guided adaptive brachytherapy for locally advanced cervical cancer: Refining bladder dose-volume parameters. Int J Radiat Oncol Biol Phys 101: 411-420, 2018

5. Mazeron R, Maroun P, Castelnau-Marchand P, Dumas I, del Campo ER, Cao K, Slocker-Escarpa A, M'Bagui R, Martinetti F, Tailleur A, et al: Pulsed-dose rate image-guided adaptive brachytherapy in cervical cancer: Dose-volume effect relationships for the rectum and bladder. Radiother Oncol 116: 226-232, 2015.

6. Zakariaee R, Hamarneh G, Brown CJ, Gaudet M, Aquino-Parsons $\mathrm{C}$ and Spadinger I: Bladder accumulated dose in image-guided high-dose-rate brachy therapy for locally advanced cervical cancer and its relation to urinary toxicity. Phys Med Biol 61: 8408-8424, 2016.

7. Harmon G, Chinsky B, Surucu M, Harkenrider M and Small W Jr: Bladder distension improves the dosimetry of organs at risk during intracavitary cervical high-dose-rate brachytherapy. Brachytherapy 15: 30-34, 2016.

8. Siavashpour Z, Aghamiri MR, Jaberi R, Manshadi HR, Ghaderi R and Kirisits C: Optimum organ volume ranges for organs at risk dose in cervical cancer intracavitary brachytherapy. J Contemp Brachytherapy 8: 135-142, 2016.

9. Nesseler JP, Charra-Brunaud C, Salleron J, Py JF, Huertas A, Meknaci E, Courrech F, Peiffert D and Renard-Oldrini S: Effect of bladder distension on doses to organs at risk in pulsed-dose-rate 3D image-guided adaptive brachytherapy for locally advanced cervical cancer. Brachytherapy 16: 976-980, 2017.

10. Siavashpour Z, Aghamiri MR, Jaberi R, ZareAkha N, Manshadi HRD, Kirisits C and Sedaghat M: A comparison of organs at risk doses in GYN intracavitary brachytherapy for different tandem lengths and bladder volumes. J Appl Clin Med Phys 17: 5-13, 2016.

11. Jamema SV, Saju S, Mahantshetty U, Pallad S, Deshpande DD, Shrivastava SK and Dinshaw KA: Dosimetric evaluation of rectum and bladder using image-based CT planning and orthogonal radiographs with ICRU 38 recommendations in intracavitary brachytherapy. J Med Phys 33: 3-8, 2008.

12. Mahantshetty U, Shetty S, Majumder D, Adurkar P, Swamidas J, Engineer R, Chopra S and Shrivastava S: Optimal bladder filling during high-dose-rate intracavitary brachytherapy for cervical cancer: a dosimetric study. J Contemp Brachytherapy 9: 112-117, 2017.

13. No authors listed: Prescribing, recording, and reporting brachytherapy for cancer of the cervix. J ICRU 13: 1-2, NP, 2013.

14. Pötter R, Tanderup K, Kirisits C, de Leeuw A, Kirchheiner K, Nout R, Tan LT, Haie-Meder C, Mahantshetty U, Segedin B, et al: The EMBRACE II study: The outcome and prospect of two decades of evolution within the GEC-ESTRO GYN working group and the EMBRACE studies. Clin Transl Radiat Oncol 9: 48-60, 2018.

15. Pecorelli S: Revised FIGO staging for carcinoma of the vulva, cervix, and endometrium. Int J Gynaecol Obstet 105: 103-104, 2009.

16. Gay HA, Barthold HJ, O'Meara E, Bosch WR, El Naqa I, Al-Lozi R, Rosenthal SA, Lawton C, Lee WR, Sandler H, et al: Pelvic normal tissue contouring guidelines for radiation therapy: A radiation therapy oncology group consensus panel atlas. Int $\mathrm{J}$ Radiat Oncol Biol Phys 83: e353-e362, 2012.

17. Fowler JF: Review: Total doses in fractionated radiotherapy-implications of new radiobiological data. Int J Radiat Biol Relat Stud Phys Chem Med 46: 103-120, 1984. 
18. Bentzen SM and Joiner MC: The linear-quadratic approach in clinical practice. In: Basic Clinical Radiobiology. Joiner MC and van der Kogel A (eds). 4th edition. CRC Press, Boca Raton, FL, pp120-134, 2009.

19. Chen AP, Setser A, Anadkat MJ, Cotliar J, Olsen EA, Garden BC and Lacouture ME: Grading dermatologic adverse events of cancer treatments: The common terminology criteria for adverse events version 4.0. J Am Acad Dermatol 67: 1025-1039, 2012.

20. Otter S, Coates A, Franklin A, Cunningham M and Stewart A: Improving dose delivery by adding interstitial catheters to fixed geometry applicators in high-dose-rate brachytherapy for cervical cancer. Brachytherapy 17: 580-586, 2018.

21. Sharma AD, Poddar J, Suryanarayan KU, Shah SP, Parikh A, Mehta V, Phys M, Kumar T and Phys M: Dosimetric analysis of the effects of the bladder volume on organs at risk (OAR) in high-dose-rate intracavitary brachytherapy in carcinoma cervix-an institutional study. J Contemp Brachytherapy 10: 26-31, 2018.

22. Liao Y, Dandekar V, Chu JC, Turian J, Bernard D and Kiel K: Reporting small bowel dose in cervix cancer high-dose-rate brachytherapy. Med Dosim 41: 28-33, 2016.

23. Damato AL, Buzurovic I, Bhagwat MS, Cormack RA, Devlin PM, Friesen S, Hansen J, Lee LJ, Manuel MM, Cho LP, et al: The value of systematic contouring of the bowel for treatment plan optimization in image-guided cervical cancer high-dose-rate brachytherapy. Brachytherapy 16: 579-585, 2017.
24. Lee S, Rodney E, Traughber B, Biswas T, Colussi V and Podder T: Evaluation of interfractional variation of organs and displacement of catheters during high-dose-rate interstitial brachytherapy for gynecologic malignancies. Brachytherapy 16: 1192-1198, 2017

25. Tan LT, Potter R, Sturdza A, Fokdal L, Haie-Meder C, Schmid M, Gregory D, Petric P, Jurgenliemk-Schulz I, Gillham C, et al: Change in patterns of failure after image-guided brachytherapy for cervical cancer: Analysis from the RetroEMBRACE study. Int J Radiat Oncol Biol Phys 104: 895-902, 2019.

26. Zaffino P, Pernelle G, Mastmeyer A, Mehrtash A, Zhang H, Kikinis R, Kapur T and Francesca Spadea M: Fully automatic catheter segmentation in MRI with 3D convolutional neural networks: Application to MRI-guided gynecologic brachytherapy. Phys Med Biol 64: 165008, 2019.

(i)(9) This work is licensed under a Creative Commons Attribution-NonCommercial-NoDerivatives 4.0 International (CC BY-NC-ND 4.0) License. 